\title{
Lost in translation: returning germline genetic results in genome-scale cancer research
}

Amber L. Johns' ${ }^{1}$, Skye H. McKay ${ }^{1}$, Jeremy L. Humphris ${ }^{1}$, Mark Pinese', Lorraine A. Chantrill ${ }^{1}, 13,14$, R. Scott Mead ${ }^{1,18,19}$, Katherine Tucker ${ }^{7}$, Lesley Andrews ${ }^{7}$, Annabel Goodwin ${ }^{16}$, Conrad Leonard ${ }^{6}$, Hilda A. High ${ }^{17}$, Katia Nones ${ }^{6}$, Ann-Marie Patch ${ }^{6}$, Neil D. Merrett ${ }^{11,15}$, Nick Pavlakis ${ }^{20}$, Karin S. Kassahnn ${ }^{8}$, Jaswinder S. Samra ${ }^{5}$, David K. Miller ${ }^{22}$, David K. Chang 1,9,10,11,12, Marina Pajic ${ }^{1}$, Australian Pancreatic Cancer Genome Initiative, John V. Pearson ${ }^{6}$, Sean M. Grimmond ${ }^{21}$, Nicola Waddell ${ }^{6}$, Nikolajs Zeps ${ }^{2,3}$, Anthony J. Gill ${ }^{1,4}$ and Andrew V. Biankin $1,10,12,23^{*}$

\begin{abstract}
Background: The return of research results (RoR) remains a complex and well-debated issue. Despite the debate, actual data related to the experience of giving individual results back, and the impact these results may have on clinical care and health outcomes, is sorely lacking. Through the work of the Australian Pancreatic Cancer Genome Initiative (APGI) we: (1) delineate the pathway back to the patient where actionable research data were identified; and (2) report the clinical utilisation of individual results returned. Using this experience, we discuss barriers and opportunities associated with a comprehensive process of RoR in large-scale genomic research that may be useful for others developing their own policies.

Methods: We performed whole-genome $(n=184)$ and exome $(n=208)$ sequencing of matched tumour-normal DNA pairs from 392 patients with sporadic pancreatic cancer (PC) as part of the APGI. We identified pathogenic germline mutations in candidate genes $(n=130)$ with established predisposition to PC or medium-high penetrance genes with well-defined cancer associated syndromes or phenotypes. Variants from candidate genes were annotated and classified according to international guidelines. Variants were considered actionable if clinical utility was established, with regard to prevention, diagnosis, prognostication and/or therapy.

Results: A total of 48,904 germline variants were identified, with 2356 unique variants undergoing annotation and in silico classification. Twenty cases were deemed actionable and were returned via previously described RoR framework, representing an actionable finding rate of $5.1 \%$. Overall, $1.78 \%$ of our cohort experienced clinical benefit from RoR.

Conclusion: Returning research results within the context of large-scale genomics research is a labour-intensive, highly variable, complex operation. Results that warrant action are not infrequent, but the prevalence of those who experience a clinical difference as a result of returning individual results is currently low.
\end{abstract}

Keywords: Genomic data, Return of results, Research ethics, Whole-genome sequencing

\footnotetext{
* Correspondence: andrew.biankin@glasgow.ac.uk

${ }^{1}$ Cancer Research Program, Garvan Institute of Medical Research, Kinghorn

Cancer Centre, Sydney, Australia

${ }^{10}$ West of Scotland Pancreatic Unit, Glasgow Royal Infirmary, Glasgow, UK

Full list of author information is available at the end of the article
} 


\section{Background}

The advent of high throughput and more rapid genomic technologies offers an extraordinary opportunity to further our understanding of the contribution of genetic variation in complex diseases such as cancer. Considerable international efforts spanning the past decade have mapped the genomes of large cohorts of cancer patients and provided insights into prevention, early detection and treatment of cancer [1, 2]. These efforts continue to accelerate rapidly on a technical, scientific and methodological level with increased capacity and breadth of cases studied. Such work is shedding new light on what effect these genetic events have on clinical phenotypes, such as how specific genetic features relate to patterns of metastasis and treatment response. Although comprehensive catalogues of mutations across a wide range of cancers has provided us with foundational knowledge, considerable challenges remain with respect to implementing this knowledge within existing clinical frameworks.

Whether it is appropriate to give individually relevant research results back to participants in research studies, or so-called return of individual results (RoR), has emerged broadly as a complex and contentious issue that remains fervently debated [3-8]. Nevertheless, several jurisdictions have agreed that genomic results, which meet scientifically accepted criteria, should be disclosed through appropriately developed and approved frameworks within a research context [9-11]. In addition, it has been reported in the cancer setting that participants express great desire to have important research results reported to them directly and also to their relatives and spouses if results become available after the participant is deceased [12]. The myriad commentaries and reports describing approaches to RoR have focused on key upstream components such as the interpretation and classification of variants for clinical significance, the frequency and types of findings that should be returned, and with whom the responsibility of returning results lies. Despite the debate, actual data related to the experience of giving individual results back, and the impact these results may have on clinical care and health outcomes, are sorely lacking. Furthermore, it has consistently been commented that policy for RoR in research settings is required and there is a call for real approaches and outcomes of RoR to be widely shared, compared and evaluated [3, 9].

The RoR process is further complicated within largescale genome research where the premise of such research involves the sharing of samples and data across international borders and broad accessibility of these large datasets to the research community. The diverse legal, ethical and cultural norms implicit in international studies requires careful navigation across different jurisdictions and therefore harmonisation is a vital goal.
Through the prospective observational cohort design of the Australian Pancreatic Cancer Genome Initiative (APGI), we present our real-world experience of the RoR process in a contemporary research setting, detailing the trajectory from recruitment to clinical utilisation. Using these data, we aim to: (1) delineate the pathway back to the patient where actionable research data were identified in the germline; and (2) report the clinical utilisation of individual results returned. Through this we will explore the barriers and opportunities associated with a comprehensive process of RoR in large-scale international genomic research and provide a commentary on how the pragmatic approach adopted in Australia can be potentially generalised to other jurisdictions.

\section{Methods}

\section{Study population}

Patients were recruited through participating institutions of the APGI between 2009 and 2013 (http://www. pancreaticcancer.net.au) as part of the International Cancer Genome Consortium (ICGC; http://www.icgc.org). Samples were contributed by third-party international collaborators as part of the Pancreatic ICGC effort. Prospective participants were provided with information that detailed the possibility of finding genetic variations that may be of relevance to them or their family members and that such findings may not be limited to their diagnosis of pancreatic cancer (PC). The consent process allowed participants the choice to 'opt in' to have individual results communicated, and given the short survival of many individuals with $\mathrm{PC}$, the choice of to whom else they may be communicated. Ethical approval was obtained from the human research ethics committee at each participating site. All participants provided written informed consent upon entry to the study.

\section{Pathway for RoR process}

The Australian National Statement on ethical conduct in Human Research (2007) section 3.5.1 states 'Where research may discover or generate information of potential importance to the future health of participants, or their blood relatives, researchers must prepare and follow an ethically defensible plan to disclose or withhold that information'. Given this obligation in Australia, a previously described framework was utilised in this study [13], which employs a context-dependent approach and enacts a category-based system for the characterisation of research findings as endorsed by others [14, 15]. The central components of the framework are informed consent, clinical utility, multidisciplinary (MDT) review, communicability and follow-up. Communicability considers the practicality of communicating results, the circumstances of the participant and treating clinician and how best the results could be communicated. Wherever 
allowable, results are to be communicated to the clinical care provider or treating medical team for their consideration (Additional file 1). The overall process is outlined in Fig. 1. Candidate genes were selected after review of the published literature and the Online Mendelian Inheritance in Man (OMIM) database. The list $(\mathrm{n}=130$, Additional file 2) includes genes with established risk for PC, genes with a cancer-related syndrome or a well-characterised solid organ or haematological cancer phenotype $[6,16,17]$. Considering both the practical and ethical aspects, the focus was strictly on cancer-related genes as these were related to the research indication. This framework was approved by each institutional Human Research Ethics Committee and data recorded by research personnel at each step (Fig. 1).

\section{Interpretation and classification of variants}

The genomic coordinates of germline variants were annotated for gene consequence with ensembl v75 [18].

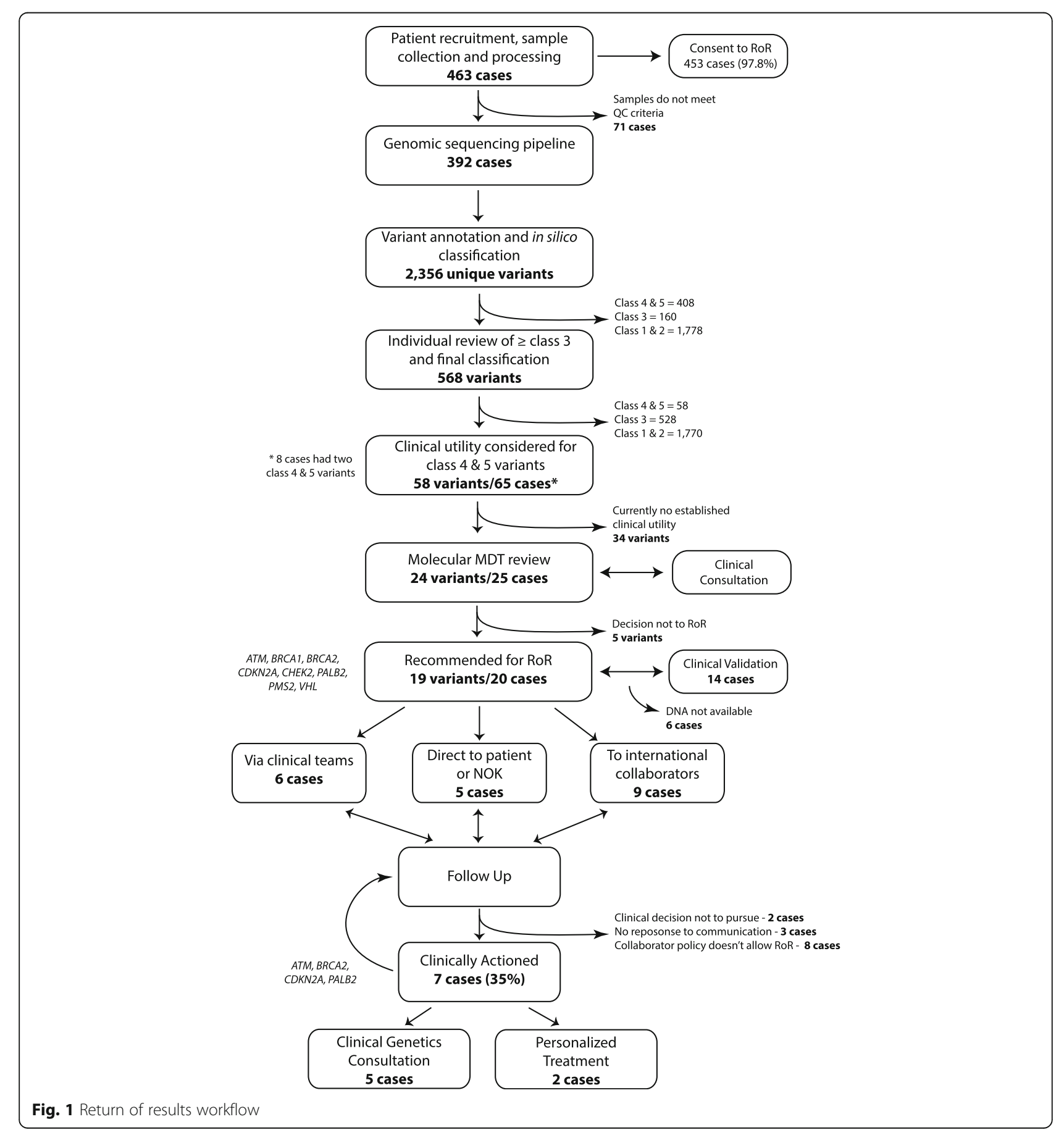


The effect of missense variants was predicted using polyphen2 [19], SIFT [20], CADD [21], VEST3 [22], alignGVGD [23], MutationTaster [24], phyloP [25] and phastCons [26]. Allele frequency in the general population was obtained from the 1000 Genomes Project (http://www.internationalgenome.org/), Exome Aggregation Consortium (http://exac.broadinstitute.org/ about) [27] and dbSNPv141 (http://www.ncbi.nlm.nih.gov/SNP). The results were compiled and variants ranked using a five-tiered schemata adapted from international guidelines $[28,29]$ (class $5=$ pathogenic, class $4=$ likely pathogenic, class $3=$ uncertain significance, class $2=$ probably no pathogenicity, $1=$ no pathogenicity, Additional file 3). Variants class 3 and above were further examined in the published literature and inherited mutation databases including ClinVar (http:// www.ncbi.nlm.nih.gov/clinvar), HGMD professional [30], OMIM and locus-specific databases (InSiGHT [28] and Leiden open variation databases [31]) before being assigned a final consensus class. Variants characterised as Class 4 or 5 (pathogenic or likely pathogenic) in genes with established clinical utility were triaged to a Molecular Multidisciplinary Team Meeting (MolMDT). The full body of variant evidence was presented to the MolMDT for evaluation and considered established clinical guidelines. These were then reviewed in the context of the individual patient and a formal report issued documenting evidence-based decision-making (Additional file 4). All cases involved consultation with clinical teams or collaborators prior to dissemination, for guidance where needed and to offer the opportunity to engage with the MolMDT discussion. Participants and clinical teams were followed up in accordance with standard APGI clinical protocols and at intervals post result communication (Fig. 1).

\section{Results}

A total of 463 patients with clinically sporadic PC were recruited to the study between 2009 and 2013. Of the participants, $97.8 \%(\mathrm{n}=453)$ indicated via the consent process that they wished to have results returned to them or to their designee. Of the cases, 392 (84\%) satisfied project-specific technical and quality essentials, such as minimum sample weight and tumour cellularity, permitting entry into the sequencing pipeline. We performed whole-genome $(n=184)$ and exome $(n=208)$ sequencing of matched tumour-normal DNA pairs from eligible patients to an average depth of $65 \times$ (tumour) and $35 \times$ (germline) under conditions previously described by this group [1,32,33]. No patient had an established hereditary cancer syndrome at enrolment. The median age at diagnosis was 67 years and the age range was 33-90 years. A total of 48,904 germline variants were identified, of which 2356 were unique in 130 candidate cancer predisposition genes. Participants had a median of 205 variants each, in the range of 2-633 variants each across the cohort. A total of $568(24 \%)$ variants were ranked as class 3 or above and underwent additional review to assess presence in published literature and inherited mutation databases [30, 34]. Fifty-eight variants $(2.5 \%)$ in 32 genes remained classified as pathogenic (Class 4 or 5) and were reported in 65 different patients. Upon final classification, 24 variants in genes with established clinical utility were reviewed at the MolMDT. Review, work up and discussion of each variant took an estimated average of 5 working hours of personnel time. Following MolMDT review 19 pathogenic variants reported in 20 cases were recommended for return. The characteristics of cases returned are described in Table 1. Cases with available sample $(\mathrm{n}=14)$ proceeded to independent validation through an accredited laboratory issuing a clinical report and all mutations were successfully validated. In cases where findings could not be validated as no residual germline tissue was available, preliminary results and recommendations from the MolMDT were returned to collaborators to pursue. In keeping with the low survival rate of $\mathrm{PC}$, ten patients $(50 \%)$ were deceased at the time results were available, four patients (20\%) were alive with disease and/or undergoing active treatment or monitoring for their disease, five $(25 \%)$ patients were discharged from primary specialist care and one (5\%) was lost to follow-up.

Observing the guidelines in the established RoR pathway results were disseminated considering context as follows: directly to the principal collaborator where samples were contributed by third parties $(n=9)$; through clinical teams where patients were undergoing active treatment or monitoring $(n=6)$; direct to patient or family members via letter where patients were deceased or no longer receiving active specialist care $(n=5)$.

Upon follow-up to date, seven cases (35\%) have been clinically actioned, defined as consultation and ongoing management within a clinical genetics service, and/or having an influence on clinical decision-making for active clinical care. Specifically, two cases with BRCA2 mutations were switched to third-line therapy with mitomycin $\mathrm{C}$. The time lapse from result dissemination to action was in the range of 59-626 days.

The residual cases not defined as actioned were categorised as follows: no allowance for RoR in collaborator policy $(\mathrm{n}=8)$; clinical decision not to pursue $(\mathrm{n}=2)$; and non-response to communication $(n=3)$.

\section{Discussion}

In this report, we describe the longitudinal process of returning germline research results in a cancer context via established mechanisms. These data allow us to illustrate a range of clinical and infrastructural challenges, 


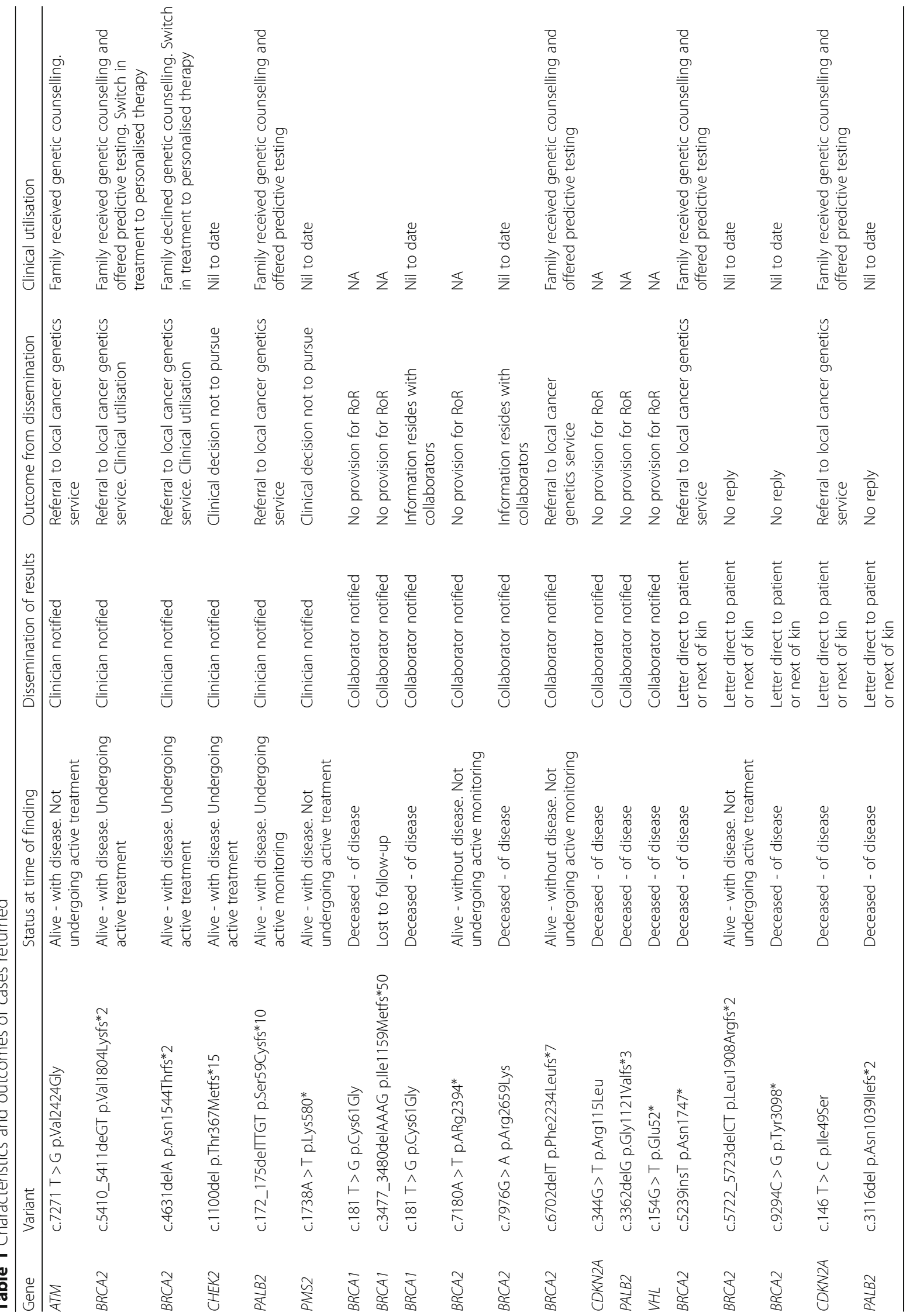


and practicability, best explored along the complex continuum of RoR. It is to the best of our knowledge the first to report this within a contemporary cancer genomics setting over a protracted period of time.

Overall, 19 pathogenic variants in eight candidate cancer genes in 20 patients were deemed actionable. This represents an actionable finding rate of $5.1 \%(n=392)$ in our cohort. Seven cases were actioned, resulting in $1.78 \%(\mathrm{n}=392)$ of the cohort experiencing a clinical benefit from returning research results. Although this number appears low, it is important to note that 63\% (n $=7 / 11$ ), of participants or family members who actually received information, actioned the finding. This is significant given that PC has a high rate of mortality and that $50 \%$ of participants were deceased at the time of return. This high rate of action establishes the importance of RoR to participants and family members and the potential benefit it may present.

Reports of actionable finding rates have been shown to be diverse and dependant on the disease setting and specific genes assessed [35, 36]. Moreover, these actionability rates are often theoretically based and defined using variant classification in absence of detail surrounding clinical utility and communicability in the context of the individual patient.

In our experience, the overall process of managing RoR was a resource-demanding activity. The requirements for developing extensive infrastructure required for evaluating genetic findings, disseminating results and performing detailed follow-up was labour-intensive, costly and time-consuming. It also required the input and coordination of a MDT consisting of both research and clinical personnel, including but not limited to: genetic pathologists; clinical geneticists; oncologists; surgeons; genetic counsellors; and bioinformaticians. Significant time and cost considerations were experienced with approximately 5 person-hours on average to review each variant prior to MolMDT, discussion within the MolMDT, independent confirmatory testing, re-contact of next of kin, and recording and disseminating paperwork. Moreover, many of these costs could not be accurately considered from the outset due to lack of guidelines on how to formally manage such a process at the time and the intrinsic unpredictability of RoR. Budgetary and personnel requirements for RoR need to be considered within the context of the individual project at the design stage alongside those for sequencing and analysis.

Due to the diversity of results and of individual patient situations, it was imperative to allow flexibility in the method of communication. Forty percent of cases where notification was by written letter were actioned and lower rates of response to letters is reported [37, 38]. Letters to next of kin were employed as the mode of communication where participants were deceased $(55 \%$ of the cohort,
Table 1), as this represented the only remaining and appropriate outlet to communicate with them. Letters may have been emotionally confronting when received by the next of kin in these situations, especially if they were unaware of the research. Returning results to relatives of deceased participants is not currently widespread practice [39], and while procedures were in place for deceased participant cases, it is recommended that participants are made aware of this possibility and asked to discuss it with their relatives if possible while alive. Australian Privacy law [40] does not address the use of information from deceased persons. However, an important amendment to the privacy act [41] allows for communication with family members without consent of a person under their care if a medical practitioner feels there is a risk of harm to them arising from knowledge about that person. Greater action rates $(66 \%$, Table 1) were experienced where treating clinicians were notified and the participants and or family were informed face-to-face by the clinical team. This existing relationship allows for these often-complex discussions to take place in an environment where information can be reinforced and relevance put into perspective for clinical decision-making. Engaging treating clinicians in the MolMDT process prior to dissemination can facilitate a smooth transition into necessary clinical pathways or therapeutic actions, as they are armed with paperwork necessary for referrals of further genetic testing or other relevant supporting information. In summary, our experiences demonstrate that result dissemination needs to be considered in the context of the participant and their family at that specific point in time.

Returning results discovered through the work of international scientific research consortia adds a layer of complexity not routinely experienced by research studies in the past. There is currently no guidance for international consortia, and in most instances the responsibility for RoR is delegated to the local level through consortia developed policies. In our study, $45 \%$ of actionable cases were from international collaborators (Table 1) and the applicability of our RoR process was restricted due to collaborators local polices, consent parameters and country-specific guidelines. Only one case could be confirmed as actioned. Our experiences highlight the need for a level of harmonisation of RoR approaches globally. Furthermore, the value and suitability of individually developed consortia-level policies as the primary management tool for RoR will inevitably be bought into question as genome sequencing on a large scale continues to advance. Future approaches could include integration of consortium level oversight while allowing for necessary local autonomy.

Despite the vast amount of effort on review and dissemination of results, the overall number of patients who experienced a clinical benefit from the return of research results in our cohort was low. Furthermore, this low action rate was experienced despite the research 
being associated with a diverse team of scientific and clinical specialties, regular participant follow-up, high participant and community engagement, a considered budget and experience with producing high quality datasets. Moreover, these findings were mostly related to well-established cancer genes routinely managed in clinical care. Our study highlights how clinicians may be expected to respond to genomic information they had not initiated, but are receiving directly or indirectly through research targeting the tumour genome. Clinicians need to be equipped and empowered to use genomic information in routine practice and concerns about genetic literacy and preparedness for genome sequencing has been voiced by clinicians themselves $[42,43]$. Further complexities arise when pathogenic mutations are detected outside of a typical disease phenotype where clear evidence based guidelines for management are less established, as illustrated by the clinical decision not to act in two cases (Table 1). Redesigning a pathway that enables RoR as part of the health system will enhance the interaction across the continuum of research and clinical care and help address the feasibility and economic issues and in turn support sustainability.

While case studies, commentaries and opinion pieces have been useful to date, the RoR process is not static. Application of RoR requires a dynamic longitudinal view of the process incorporated with outcomes. Effectively navigating the RoR process is a current global challenge and significant investment has been recently injected to analyse the ethical, social and legal effects of genomic information [44]. Reports of empirical data are crucial to ascertain the range of benefits and harms of RoR. Plenty of obstacles and challenges no doubt remain, as returning important research information to participants is a young pursuit. The significant investment in overcoming the challenges of sequencing and analysis on a large scale needs to be mirrored in RoR, as the technology of genomic sequencing itself moves into clinical care, application of important research results is fundamental to translational medicine.

\section{Conclusion}

Of our cohort, $5.1 \%$ had results that were theoretically actionable. However, only seven cases were actioned, resulting in $1.78 \%$ of the cohort of 392 participants deriving clinical benefit from RoR. Despite this low number, $63 \%$ of those who received information acted upon this information, demonstrating that RoR stands to be of significant value to participants and family members if we can work to steadily remove the clinical and procedural barriers to implementation.

\section{Additional files}

Additional file 1: APGI Return of Results Guidelines, which outline standard procedures instituted for the return of results process. (PDF $832 \mathrm{~kb}$ )

Additional file 2: Gene List, which includes the list of candidate genes selected for the RoR process. (XLSX $60 \mathrm{~kb}$ )

Additional file 3: Variant Classification Schema, which outlines the bioinformatic and final classification ranking for assessment of variants. (PDF $271 \mathrm{~kb}$ )

Additional file 4: Molecular MDT Protocol, which outlines standard operating procedures for the MoIMDT. (PDF $271 \mathrm{~kb}$ )

\section{Abbreviations}

MolMDT: Molecular multidisciplinary team meeting; PC: Pancreatic cancer; RoR: Return of results

\section{Acknowledgments}

We thank all members of the APGI for their continued collaboration (http:// www.pancreaticcancer.net.au/about-collaborators/). We also thank all our participants and their families for their involvement in this study, without whom none of this work would be possible.

Australian Pancreatic Cancer Genome Initiative

Garvan Institute of Medical Research Amber L. Johns ${ }^{1}$, Anthony J Gill 1, 5 David K. Chang ${ }^{1,22}$, Skye H. McKay', Lorraine A. Chantrill ${ }^{1,8}$, Venessa T. Chin ${ }^{1}$, Angela Chou ${ }^{1,8}$, Jeremy L. Humphris ${ }^{1}$, Marina Pajic ${ }^{1}$, Angela Steinmann", Mehreen Arshi ${ }^{1}$, Ali Drury', Danielle Froio ${ }^{1}$, Ashleigh Morgan', Paul Timpson ${ }^{1}$ David Hermann" ${ }^{1}$ Claire Vennin', Sean Warren', Mark Pinese ${ }^{1}$, Jianmin Wu' Andreia V. Pinho'. QIMR Berghofer Medical Research Institute Nicola Waddell $^{2}$, John V. Pearson², Ann-Marie Patch², Katia Nones², Felicity Newell ${ }^{2}$, Pamela Mukhopadhyay ${ }^{2}$, Venkateswar Addala², Stephen Kazakoff ${ }^{2}$, Oliver Holmes $^{2}$, Conrad Leonard ${ }^{2}$, Scott Wood ${ }^{2}$, Christina X $\mathbf{u}^{2}$. University of Melbourne, Centre for Cancer Research Sean M. Grimmond ${ }^{3}$, Oliver Hofmann ${ }^{3}$. University of QLD, IMB Peter J. Wilson ${ }^{4}$, Angelika Christ ${ }^{4}$, Tim Bruxner ${ }^{4}$. Royal North Shore Hospital Jaswinder S. Samra ${ }^{5}$, Jennifer Arena ${ }^{5}$, Nick Pavlakis ${ }^{5}$ Hilda A. High ${ }^{5}$, Anubhav Mittal ${ }^{5}$. Bankstown Hospital Ray Asghari ${ }^{6}$, Neil D. Merrett ${ }^{6}$, Darren Pavey ${ }^{6}$, Amitabha Das ${ }^{6}$. Liverpool Hospital Peter H. Cosman', Kasim Ismail', Chelsie O'Connnor ${ }^{7}$. St Vincent's Hospital Alina Stoita ${ }^{8}$, David Williams ${ }^{8}$, Allan Spigellman ${ }^{8}$. Westmead Hospital Vincent W. Lam ${ }^{9}$. Duncan McLeod ${ }^{9}$, Adnan M. Nagrial $^{1,9}$, Judy Kirk' ${ }^{9}$ Virginia James ${ }^{9}$. Royal Prince Alfred Hospital, Chris O'Brien Lifehouse James G. Kench ${ }^{10}$, Peter Grimison ${ }^{10}$, Caroline L. Cooper ${ }^{10}$, Charbel Sandroussi ${ }^{10}$, Annabel Goodwin ${ }^{7,10}$. Prince of Wales Hospital R. Scott Mead ${ }^{1,11}$, Katherine Tucker ${ }^{11}$, Lesley Andrews ${ }^{11}$. Fremantle Hospital Michael Texler ${ }^{12}$, Cindy Forest $^{12}$, Krishna P. Epari ${ }^{12}$, Mo Ballal ${ }^{12}$, David R. Fletcher ${ }^{12}$, Sanjay Mukhedkar ${ }^{12}$. St John of God Healthcare Nikolajs Zeps ${ }^{14}$, Maria Beilin ${ }^{14}$, Kynan Feeney ${ }^{14}$. Royal Adelaide Hospital Nan Q Nguyen ${ }^{15}$, Andrew R. Ruszkiewicz ${ }^{15}$, Chris Worthley ${ }^{15}$. Flinders Medical Centre John Chen ${ }^{16}$, Mark E. Brooke-Smith ${ }^{16}$, Virginia Papangelis ${ }^{16}$. Envoi Pathology Andrew D. Clouston ${ }^{17}$, Patrick Martin ${ }^{17}$. Princess Alexandria Hospital Andrew P. Barbour ${ }^{18}$, Thomas J. O'Rourke ${ }^{18}$ Jonathan W. Fawcett ${ }^{18}$, Kellee Slater ${ }^{18}$, Michael Hatzifotis ${ }^{18}$, Peter Hodgkinson ${ }^{18}$. Austin Hospital Mehrdad Nikfarjam ${ }^{19}$. Johns Hopkins Medical Institutes James R. Eshleman $^{20}$, Ralph H. Hruban ${ }^{20}$, Christopher L. Wolfgang ${ }^{20}$, Mary Hodgin ${ }^{20}$. ARC-Net Centre for Applied Research on Cancer Aldo Scarpa ${ }^{21}$, Rita T. Lawlor ${ }^{21}$, Stefania Beghelli ${ }^{21}$, Vincenzo Corbo ${ }^{21}$, Maria Scardoni ${ }^{21}$, Claudio Bassi ${ }^{21}$.

University of Glasgow Andrew V Biankin 1, 22, Peter Bailey ${ }^{22}$, Sancha Martin $^{22}$, Elizabeth A. Musgrove ${ }^{22}$, Marc D. Jones ${ }^{22}$, Craig Nourse ${ }^{22}$, Nigel B. Jamieson $^{22}$

${ }^{1}$ The Kinghorn Cancer Centre, Garvan Institute of Medical Research, 370 Victoria Street, Darlinghurst, Sydney, NSW 2010, Australia.

${ }^{2}$ QIMR Berghofer Medical Research Institute, 300 Herston Rd, Herston, QLD

4006, Australia.

${ }^{3}$ University of Melbourne, Centre for Cancer Research, Victorian

Comprehensive Cancer Centre, 305 Grattan Street, Melbourne, VIC 3000, Australia.

${ }^{4}$ Institute for Molecular Bioscience, University of QLD, St Lucia, QLD 4072,

Australia.

${ }^{5}$ Royal North Shore Hospital, Westbourne Street, St Leonards, NSW 2065, Australia.

${ }^{6}$ Bankstown Hospital, Eldridge Road, Bankstown, NSW 2200, Australia.

${ }^{7}$ Liverpool Hospital, Elizabeth Street, Liverpool, NSW 2170, Australia. 
${ }^{8}$ St Vincent's Hospital, 390 Victoria Street, Darlinghurst, NSW 2010, Australia. ${ }^{9}$ Westmead Hospital, Hawkesbury and Darcy Roads, Westmead, NSW 2145, Australia.

${ }^{10}$ Royal Prince Alfred Hospital, Missenden Road, Camperdown, NSW 2050, Australia.

${ }^{11}$ Prince of Wales Hospital, Barker Street, Randwick, NSW 2031, Australia.

${ }^{12}$ Fremantle Hospital, Alma Street, Fremantle, WA 6959, Australia.

${ }^{13}$ Sir Charles Gairdner Hospital, Hospital Avenue, Nedlands, WA 6009, Australia.

${ }^{14}$ St John of God Healthcare, 12 Salvado Road, Subiaco, WA 6008, Australia.

${ }^{15}$ Royal Adelaide Hospital, North Terrace, Adelaide, SA 5000, Australia.

${ }^{16}$ Flinders Medical Centre, Flinders Drive, Bedford Park, SA 5042, Australia.

${ }^{17}$ Envoi Pathology, 1/49 Butterfield Street, Herston, QLD 4006, Australia.

${ }^{18}$ Princess Alexandria Hospital, Cornwall Street \& Ipswich Road,

Woolloongabba, QLD 4102, Australia.

${ }^{19}$ Austin Hospital, 145 Studley Road, Heidelberg, VIC 3084, Australia.

${ }^{20}$ Johns Hopkins Medical Institute, 600 North Wolfe Street, Baltimore, MD

21287, USA.

${ }^{21}$ ARC-NET Center for Applied Research on Cancer, University of Verona, Via dell'Artigliere, 1937129 Verona, Province of Verona, Italy.

${ }^{22}$ Wolfson Wohl Cancer Research Centre, Institute of Cancer Sciences, University of Glasgow, Garscube Estate, Switchback Road, Bearsden, Glasgow, Scotland G61 1BD, UK

\section{Funding}

National Health and Medical Research Council of Australia (NHMRC; 631701, 535903, 427601, 1112113)

Queensland Government (NIRAP)

University of Queensland

Institute for Molecular Bioscience

Australian Government: Department of Innovation, Industry, Science and Research (DIISR)

Cancer Council NSW: (SRP06-01, SRP11-01. ICGC)

Cancer Institute NSW: (10/ECF/2-26; 06/ECF/1-24; 09/CDF/2-40; 07/CDF/1-03; 10/CRF/1-01, 08/RSA/1-15, 07/CDF/1-28, 10/CDF/2-26,10/FRL/2-03, 06/RSA11-05, 09/RIG/1-02, 10/TPG/1-04, 11/REG/1-10, 11/CDF/3-26,13ECF1-46, 13/CDF/1-01) Garvan Institute of Medical Research

Avner Nahmani Pancreatic Cancer Foundation

Jane Hemstritch in memory of Philip Hemstritch.

\section{Availability of data and materials}

The datasets generated and analysed during the current study are not publicly available due the data being germline and classified as controlled. A subset of these data is available upon application and approval from the ICGC Data Access Compliance Office (DACO): http://icgc.org/daco.

\section{Authors' contributions}

$A L J, S H M, J L H, N W, N Z, A J G, A V B$ and SMG were involved in concept and design of the study, MP, RSM, CRL, KN, AMP, KSS, DKM, MP, APGI, DKC and JVP analysed, annotated and interpreted the data set, LAC, KT, LA, AG, HAH NDM, NP and JSS were involved in acquisition and analysis of clinical data. All authors were involved in manuscript writing and final approval of the manuscript.

\section{Competing interests}

AVB has an advisory role for Cure Forward, Asta Zeneca and Celgene. NP has held advisory roles for Baxalta, Speclialsed Therapuetics, Roche, Astra Zeneca, Pfizer, Novatis, Amgen and Bayer.

DKM holds stocks in Illumina Inc.

All other authors declare they have no competing interests.

\section{Consent for publication}

Not applicable.

\section{Ethics approval and consent to participate}

Ethical approval was obtained from the human research ethics committee at each participating site. All participants provided written informed consent upon entry to the study. The study was performed in accordance with the Declaration of Helsinki.

Sydney South West Area Health Service Human Research Ethics Committee western zone (protocol number 2006/54); Sydney Local Health District
Human Research Ethics Committee (X11-0220); Northern Sydney Central Coast Health Harbour Human Research Ethics Committee (0612-251 M); Royal Adelaide Hospital Human Research Ethics Committee (091107a); Metro South Human Research Ethics Committee (09/QPAH/220); South Metropolitan Area Health Service Human Research Ethics Committee (09/324); Southern Adelaide Health Service/Flinders University Human Research Ethics Committee (167/10); Sydney West Area Health Service Human Research Ethics Committee (Westmead campus) (HREC2002/3/ 4.19); The University of Queensland Medical Research Ethics Committee (2009000745); Greenslopes Private Hospital Ethics Committee (09/34); North Shore Private Hospital Ethics Committee. Johns Hopkins Medicine Institutional Review Board (NA00026689). University of Verona: approval number 1885 from the Integrated University Hospital Trust (AOUI). Ethikkommission an der Technischen Universität Dresden (Approval numbers EK30412207 and EK357112012).

\section{Publisher's Note}

Springer Nature remains neutral with regard to jurisdictional claims in published maps and institutional affiliations.

\section{Author details}

${ }^{1}$ Cancer Research Program, Garvan Institute of Medical Research, Kinghorn Cancer Centre, Sydney, Australia. ${ }^{2}$ St John of God Subiaco, Perth, Australia. ${ }^{3}$ School of Surgery, The University of Western Australia, Perth, Australia. ${ }^{4}$ Cancer Diagnosis and Pathology Group, Kolling Institute of Medical Research, Royal North Shore Hospital, Sydney Australia and University of Sydney, Sydney, Australia. ${ }^{5}$ Department of Surgery, Royal North Shore Hospital, Sydney, Australia. ${ }^{6}$ QIMR Berghofer Medical Research Institute, Brisbane, Australia. ${ }^{7}$ Hereditary Cancer Clinic, Prince of Wales Hospital, Randwick, Sydney, Australia. ${ }^{8}$ Genetic and Molecular Pathology, SA Pathology, Women's and Children's Hospital, North Adelaide, Adelaide, Australia. ${ }^{9}$ Wolfson Wohl Cancer Research Centre, Institute of Cancer Sciences, University of Glasgow, Glasgow, Scotland, UK. ${ }^{10}$ West of Scotland Pancreatic Unit, Glasgow Royal Infirmary, Glasgow, UK. " Department of Surgery, Bankstown Hospital, Eldridge Road, Bankstown, Sydney, Australia. ${ }^{12}$ South Western Sydney Clinical School, Faculty of Medicine, University of New South Wales, Liverpool, Australia. ${ }^{13}$ St Vincents Hospital, Darlinghurst, Australia. ${ }^{14}$ Western Sydney University Clinical School, Sydney, Australia. ${ }^{15}$ Division of Surgery, School of Medicine, Western Sydney University, Sydney, Australia. ${ }^{16}$ Cancer Genetics Department, Royal Prince Alfred Hospital and Liverpool Hospital, Sydney, NSW, Australia. ${ }^{17}$ Sydney Cancer Genetics, Sydney, Australia. ${ }^{18}$ Genetics Department, SEALS Pathology, Prince of Wales Hospital, Randwick, Sydney, Australia. ${ }^{19}$ School of Medicine, University of New South Wales, Sydney, Australia. ${ }^{20}$ Department of Medical Oncology, Royal North Shore Hospital and Faculty of Medicine, University of Sydney, Sydney, Australia. ${ }^{21}$ University of Melbourne, Parkville, Australia. ${ }^{22}$ Illumina Inc, 5200 Illumina Way, San Diego, CA 92122, USA. ${ }^{23}$ Wolfson Wohl Cancer Research Centre, Institute of Cancer Sciences, University of Glasgow, Garscube Estate, Switchback Road, Bearsden, Glasgow, UK.

\section{Received: 8 February 2017 Accepted: 12 April 2017}

Published online: 28 April 2017

\section{References}

1. Biankin AV, Waddell N, Kassahn KS, Gingras MC, Muthuswamy LB, Johns AL, et al. Pancreatic cancer genomes reveal aberrations in axon guidance pathway genes. Nature. 2012;491(7424):399-405.

2. International Cancer Genome Consortium, Hudson TJ, Anderson W, Artez A, Barker AD, Bell C, et al. International network of cancer genome projects. Nature. 2010;464(7291):993-8.

3. Wolf SM. Return of individual research results and incidental findings: facing the challenges of translational science. Annu Rev Genomics Hum Genet. 2013;14:557-77.

4. McGuire AL, Joffe $S$, Koenig BA, Biesecker BB, McCullough LB, Blumenthal-Barby JS, et al. Point-counterpoint. Ethics and genomic incidental findings. Science. 2013;340(6136):1047-8.

5. Wolf SM, Annas GJ, Elias S. Point-counterpoint. Patient autonomy and incidental findings in clinical genomics. Science. 2013;340(6136): 1049-50. 
6. Green RC, Berg JS, Grody WW, Kalia SS, Korf BR, Martin CL, et al. ACMG recommendations for reporting of incidental findings in clinical exome and genome sequencing. Genet Med. 2013;15(7):565-74.

7. Biesecker LG. Incidental variants are critical for genomics. Am J Hum Genet. 2013;92(5):648-51.

8. ACMG Board of Directors. ACMG policy statement: updated recommendations regarding analysis and reporting of secondary findings in clinical genome-scale sequencing. Genet Med. 2015;17(1):68-9.

9. Jarvik GP, Amendola LM, Berg JS, Brothers K, Clayton EW, Chung W, et al. Return of genomic results to research participants: the floor, the ceiling, and the choices in between. Am J Hum Genet. 2014;94(6):818-26.

10. Weiner C. Anticipate and communicate: Ethical management of incidental and secondary findings in the clinical, research and direct-to-consumer contexts (December 2013 report of the Presidential Commission for the Study of Bioethical Issues). Am J Epidemiol. 2014;180(6):562-4.

11. National Heart, Lung, and Blood Institute working group, Fabsitz RR, McGuire A, Sharp RR, Puggal M, Beskow M, et al. Ethical and practical guidelines for reporting genetic research results to study participants: updated guidelines from a National Heart, Lung, and Blood Institute working group. Circ Cardiovasc Genet. 2010;3(6):574-80.

12. Breitkopf CR, Petersen GM, Wolf SM, Chaffee KG, Robinson ME, Gordon DR, et al. Preferences regarding return of genomic results to relatives of research participants, including after participant death: empirical results from a cancer biobank. J Law Med Ethics. 2015;43(3):464-75.

13. Johns AL, Miller DK, Simpson SH, Gill AJ, Kassahn KS, Humphris JL, et al. Returning individual research results for genome sequences of pancreatic cancer. Genome Med. 2014;6(5):42.

14. Berg JS, Amendola LM, Eng C, Van Allen E, Gray SW, Wagle N, et al. Processes and preliminary outputs for identification of actionable genes as incidental findings in genomic sequence data in the Clinical Sequencing Exploratory Research Consortium. Genet Med. 2013;15(11):860-7.

15. Berg JS, Khoury MJ, Evans JP. Deploying whole genome sequencing in clinical practice and public health: meeting the challenge one bin at a time. Genet Med. 2011:13(6):499-504.

16. Roberts NJ, Norris AL, Petersen GM, Bondy ML, Brand R, Gallinger S, et al. Whole genome sequencing defines the genetic heterogeneity of familial pancreatic cancer. Cancer Discov. 2016;6(2):166-75.

17. Rahman N. Realizing the promise of cancer predisposition genes. Nature. 2014;505(7483):302-8.

18. McLaren W, Pritchard B, Rios D, Chen Y, Flicek P, Cunningham F. Deriving the consequences of genomic variants with the Ensembl API and SNP Effect Predictor. Bioinformatics. 2010;26(16):2069-70.

19. Adzhubei I, Jordan DM, Sunyaev SR. Predicting functional effect of human missense mutations using PolyPhen-2. Current protocols in human genetics/ editorial board, Jonathan L. Haines ... [et al.]. Jan 2013;Chapter 7:Unit7 20.

20. Kumar P, Henikoff S, Ng PC. Predicting the effects of coding non-synonymous variants on protein function using the SIFT algorithm. Nat Protoc. 2009;4(7):1073-81.

21. Kircher M, Witten DM, Jain P, O'Roak BJ, Cooper GM, Shendure J. A genera framework for estimating the relative pathogenicity of human genetic variants. Nat Genet. 2014:46(3):310-5.

22. Carter H, Douville C, Stenson PD, Cooper DN, Karchin R. Identifying Mendelian disease genes with the variant effect scoring tool. BMC Genomics. 2013;14 Suppl 3:S3.

23. Mathe $E$, Olivier M, Kato S, Ishioka C, Hainaut $P$, Tavtigian SV. Computational approaches for predicting the biological effect of p53 missense mutations: a comparison of three sequence analysis based methods. Nucleic Acids Res. 2006;34(5):1317-25.

24. Schwarz JM, Rodelsperger C, Schuelke M, Seelow D. MutationTaster evaluates disease-causing potential of sequence alterations. Nat Methods. 2010;7(8):575-6

25. Pollard KS, Hubisz MJ, Rosenbloom KR, Siepel A. Detection of nonneutral substitution rates on mammalian phylogenies. Genome Res. 2010;20(1):110-21.

26. Siepel A, Bejerano G, Pedersen JS, Hinrichs AS, Hou M, Rosenbloom K, et al. Evolutionarily conserved elements in vertebrate, insect, worm, and yeast genomes. Genome Res. 2005;15(8):1034-50.

27. Lek M, Karczewski KJ, Minikel EV, Samocha KE, Banks E, Fennell T, et al. Analysis of protein-coding genetic variation in 60,706 humans. Nature. 2016;536(7616):285-91.

28. Thompson BA, Spurdle AB, Plazzer JP, Greenblatt MS, Akagi K, Al-Mulla F, et al. Application of a 5-tiered scheme for standardized classification of 2,360 unique mismatch repair gene variants in the InSiGHT locus-specific database. Nat Genet. 2014:46(2):107-15.

29. Richards S, Aziz N, Bale S, Bick D, Das S, Gastier-Foster J, et al. Standards and guidelines for the interpretation of sequence variants: a joint consensus recommendation of the American College of Medical Genetics and Genomics and the Association for Molecular Pathology. Genet Med. 2015;17(5):405-24.

30. Stenson PD, Mort M, Ball EV, Howells K, Phillips AD, Thomas NS, et al. The Human Gene Mutation Database: 2008 update. Genome Med. 2009;1(1):13.

31. Fokkema IFAC, Taschner PEM, Schaafsma GCP, Celli J, Laros JFJ, den Dunnen JT. LOVD v. 2.0: the next generation in gene variant databases. Hum Mutat. 2011:32(5):557-63.

32. Waddell N, Pajic M, Patch AM, Chang DK, Kassahn KS, Bailey P, et al. Whole genomes redefine the mutational landscape of pancreatic cancer. Nature 2015;518(7540):495-501.

33. Bailey P, Chang DK, Nones $K$, Johns AL, Patch AM, Gingras MC, et al. Genomic analyses identify molecular subtypes of pancreatic cancer. Nature. 2016;531(7592):47-52.

34. ClinVar. https://www.ncbi.nlm.nih.gov/clinvar/. Accessed June 2010.

35. Lawrence L, Sincan M, Markello T, Adams DR, Gill F, Godfrey R, et al. The implications of familial incidental findings from exome sequencing: the $\mathrm{NIH}$ Undiagnosed Diseases Program experience. Genet Med. 2014;16(10):741-50.

36. Dorschner MO, Amendola LM, Turner EH, Robertson PD, Shirts BH, Gallego CJ, et al. Actionable, pathogenic incidental findings in 1,000 participants' exomes. Am J Hum Genet. 2013:93(4):631-40.

37. Wakefield CE, Thorne H, Kirk J, Niedermayr E, Doolan EL, Katheleen Cunningham National Consortium for Research, et al. Improving mutation notification when new genetic information is identified in research: a trial of two strategies in familial breast cancer. Genet Med. 2013;15(3):187-94.

38. Forrest LE, Young MA. Clinically significant germline mutations in cancercausing genes identified through research studies should be offered to research participants by genetic counselors. J Clin Oncol. 2016;34(9):898-901.

39. Wolf SM, Branum R, Koenig BA, Petersen GM, Berry SA, Beskow LM, et al. Returning a research participant's genomic results to relatives: analysis and recommendations. J Law Med Ethics. 2015;43(3):440-63.

40. Go A. Privacy Act 1988, vol. 119. Canberra: ComLaw; 1988.

41. Australia Go. PRIVACY ACT 1988 - SECT 95AA. http://www.austlii.edu.au/au/ legis/cth/consol_act/pa1988108/s95aa.html. Accessed Nov 2016.

42. Vassy JL, Christensen KD, Slashinski MJ, Lautenbach DM, Raghavan S, Robinson JO, et al. 'Someday it will be the norm': physician perspectives on the utility of genome sequencing for patient care in the MedSeq Project. Per Med. 2015;12(1):23-32.

43. Gray SW, Hicks-Courant K, Cronin A, Rollins BJ, Weeks JC. Physicians' attitudes about multiplex tumor genomic testing. J Clin Oncol. 2014:32(13):1317.

44. NIH pumps \$15M into studies on effects of genomics information. 2016; https://www.genomeweb.com/research-funding/nih-pumps-15m-studieseffects-genomics-information 15 million. Accessed 10 May 2016.

\section{Submit your next manuscript to BioMed Central and we will help you at every step:}

- We accept pre-submission inquiries

- Our selector tool helps you to find the most relevant journal

- We provide round the clock customer support

- Convenient online submission

- Thorough peer review

- Inclusion in PubMed and all major indexing services

- Maximum visibility for your research

Submit your manuscript at www.biomedcentral.com/submit 